\title{
The effects of pulsed and continuous stimulation on vibrotactile thresholds obtained from the tongue
}

\author{
DONALD J. FUCCI and DENNIS J. ARNST \\ Hearing and Speech Sciences, Ohio University, Athens, Ohio 45701 \\ and \\ KAL M. TELAGE \\ State University of New York, Geneseo, N.Y. 14454
}

Vibrotactile thresholds were obtained from the anterior midline surface of the tongue on two groups of normal-speaking young adults by using ascending-pulsed, ascending-continuous, and descending-continuous stimulation. The $t$ test comparisons of the data showed no significant differences between ascending-pulsed and continuous thresholds; however, significant $t$ tests were obtained when comparing ascending-continuous and descending-continuous data. These results suggested that descending-continuous vibrotactile thresholds were significantly poorer than either ascending-pulsed or continuous thresholds.

Since speech is essentially a motor act consisting of highly coordinated muscle synergies, the presence of an automatic monitoring system can be precluded. Movement of the tongue within the oral cavity and its placement against the various other articulators stimulates tactile, kinesthetic, and proprioceptive components of the feedback mechanisms involved in articulation. Smith \& Henry (1967) indicated that the continuous closed-loop tracking of speech sound was not only reflected in the sound patterns of speech, but also in specific somesthetic processes related to articulatory movements. The application of the servo theory to speech production (Fairbanks, 1954; Van Riper \& Irwin, 1962; Mysak, 1966) has led investigators to explore oral feedback mechanisms and their role in the speech act.

In comparing the vibrotactile thresholds obtained from the lip and tongue, Fucci (1972) reported that speech-defective $\mathrm{Ss}$ were less sensitive to vibrotactile stimulation than were unaffected speakers. The implication of this study suggested that poor oral vibrotactile sensitivity represented an interruption of the sensory control unit necessary for proper coordination of the articulators. Furthermore, it emphasized the importance of the tactile sensory system and its contribution to the speech feedback network.

Various critical parameters affecting vibrotactile research have been reported in the literature. Verrillo (1963) found that doubling the area of the contactor used in stimulating test structures produced a $3-\mathrm{dB}$ shift in threshold. The increased sensitivity was suggested to be the result of energy summation which had occurred over the entire surface of the contactor. Verrillo (1963) also pointed out the importance of limiting the spread of transverse waves beyond the point of stimulation. This was accomplished through the use of a rigid surface containing a hole large enough to encircle the contactor with a constant gap of $1 \mathrm{~mm}$. The use of the free surround structure further substantiated contactor area as a significant stimulus parameter.

Hall, Fucci, \& Arnst (1972) studied the effects of different psychophysical methods on vibrotactile thresholds obtained from the midline surface of the tongue. No statistically significant differences were definable between a method of limits or method of adjustment using ascending or descending threshold data. An ascending, pulsed stimulus, however, was recommended for use in threshold testing to take advantage of the initial response of the receptor unit and avoid any possible adaptation effects.

As a further attempt to define appropriate methods for obtaining vibrotactile threshold data from the midline surface on the tongue, the present study was designed to compare ascending-pulsed and continuous thresholds. Secondarily, lingual thresholds were also analyzed for conditions of ascending and descending continuous stimulation.

\section{METHOD}

The Ss (age range, 18-36 years) used in the present study reported no history of speech defects and sensory or motor impairments. Fifty-two Ss were tested using an ascending procedure with pulsed and continuous stimulation. The threshold data recorded for the ascending-continuous condition were also compared with descending-continuous thresholds obtained on another group of $52 \mathrm{Ss}$.
The apparatus used in the present experiment has been described in detail in a previous publication (Fucci, 1972). The stimulus unit included a sine wave generator, frequency counter, electronic switch (designed to maintain a sine wave configuration), interval timer, amplifier, variable attenuator, and electromagnetic vibrator. The pulsed vibratory signal generated had a 50\% duty cycle (on $1 \mathrm{sec}$ and off $1 \mathrm{sec}$ ) with a rise and decay time of $100 \mathrm{msec}$. The measurement unit consisted of an accelerometer, cathode follower, microphone amplifier, and vacuum tube voltmeter. A white noise generator was used for auditory masking at 100-110 dB SPL.

Each $\mathbf{S}$ was seated in a dental chair and extended his tongue between two sterilized plastic disks which clamped the structure comfortably and without affecting normal circulation. The stimulus was applied to the dorsal anterior midline surface of the tongue through a free-surround disk containing a hole with a diameter $2 \mathrm{~mm}$ greater than that of the contactor. Contactor area was $.128 \mathrm{~cm}^{2}$ (diam $\left.=.404 \mathrm{~cm}\right)$ and was within a range reported by Verrillo (1962, 1963).

The vibrator was mounted on an adjustable arm and could be lowered into position. In order to maintain constant pressure of the vibrator on the tongue for all Ss, the unit was lowered $1 \mathrm{~mm}$ beyond minimum contact with the tongue. This was accomplished by using a simple circuit consisting of an ohmmeter with its negative wire connected to the base of the electromagnetic vibrator and its positive wire between S's thumb and forefinger.

Lingual vibrotactile thresholds were obtained at $300 \mathrm{~Hz}$ for all Ss using a method of limits with either an ascending-pulsed and continuous or descending-continuous series of stimulation. Ss were required to depress an indicator button as soon as they either detected or ceased to detect the stimulus under the appropriate condition. A median of $3-\mathrm{mV}$ readings was accepted as the lingual threshold and was later changed to displacement in microns with a standard $g$ formula for acceleration conversion.

\section{RESULTS}

Data in microns were averaged across $S s$ for the ascending-pulsed, a s cending - continuous, and descending-continuous conditions. These results appear in Table 1 . The mean descending-continuous threshold appears to be greater than either of the other thresholds. Ascending thresholds for the pulsed and continuous conditions were submitted to a $t$ test 
Table 1

Means and Standard Deviations for Each Group of Threshold Data

\begin{tabular}{lcc}
\hline \multicolumn{1}{c}{ Threshold Data } & $\begin{array}{c}\text { Mean } \\
\text { (in Microns) }\end{array}$ & SD \\
\hline Ascending-Pulsed & .176 & .135 \\
Ascending-Continuous & .193 & .110 \\
Descending-Continuous & .562 & .132 \\
\hline
\end{tabular}

Table 2

Summary of $t$ Test Comparisons of Threshold Data

\begin{tabular}{lc}
\multicolumn{1}{c}{ Comparison } & $\mathrm{t}$ Test \\
\hline $\begin{array}{l}\text { Ascending-Pulsed } \\
\text { vs }\end{array}$ & 1.209 \\
$\begin{array}{l}\text { Ascending-Continuous } \\
\text { Ascending-Continuous } \\
\text { vs }\end{array}$ & \\
Descending-Continuous & $15.534^{*}$ \\
\hline
\end{tabular}

$* p>.01$

for related measures with the alpha level set at .01. No significant difference was found between these two groups of threshold data. The ascending-continuous thresholds were compared with the descending-continuous data obtained from the second group of Ss using a test for independent means. The alpha level was again set at .01. A statistically significant difference was found to exist between these two sets of data, suggesting that obtaining vibrotactile thresholds using descending continuous stimulation produces different thresholds than when using an ascending pulsed or ascending continuous testing series. A summary of the $t$ test comparisons appears in Table 2.

Based on the results of the statistical analysis, it appears that descending-continuous vibrotactile stimulation of the tongue leads to less sensitive thresholds. Hall et al (1972) have previously established that ascending- or descending-pulsed stimulation using a method of limits or method of adjustment produces thresholds which are not significantly different. The present study furthers the earlier investigation by suggesting that the critical parameter affecting vibrotactile thresholds obtained from the tongue is continuous vs pulsed stimulation. Furthermore, it indicates that more sensitive thresholds can be obtained when using pulsed stimulation. This result is similar to those reported by Hirsh (1952) in which auditory thresholds for interrupted tones are lower (i.e., more sensitive) than those obtained with a continuous tone.

In conclusion, it can be stated that vibrotactile thresholds obtained from the midline surface on the tongue are affected by the type of stimulation used. The fact that continuous thresholds are less sensitive when using a descending series provides some preliminary basis for investigation of the adaptation effects in this area of research.

\section{REFERENCES}

FAIRBANKS, G. Systematic research in experimental phonetics. Journal of Speech \& Hearing Disorders, 1954, 19, 133-140.

FUCCI, D. J. Oral vibrotactile sensation: An evaluation of normal and defective speakers. Journal of Speech \& Hearing Research, 1972, 15, 179-184.

HALL, D. E., FUCCI, D. J., \& ARNST, D J. Vibrotactile stimulation: An investigation of psychophysical methods for establishing threshold. Perceptual \& Motor Skills, 1972, 34, 891-898.

HIRSH, I. J. The measurement of hearing. New York: McGraw-Hill, 1952

MYSAK, E. A servo model for speech therapy. Journal of Speech \& Hearing Disorders, 1959, 24, 144-149.

SMITH, K. U. \& HENRY, J. P. Cybernetic foundations for rehabilitation. American Journal of Physical Medicine, 1967, 46, 379-467.

VAN RIPER, C., \& IRWIN, J. Voice and articulation. Englewood Cliffs, N.J: Prentice-Hall, 1962

VERRILLO, R. T. Investigation of some parameters of the cutaneous threshold for vibration. Journal of the Acoustical Society of America, 1962,34 , 1768-1773.

VERRILLO, R. T. Effect of contactor area on the vibrotactile threshold. Journal of the Acoustical Society of America, 1963, $35,1962-1966$. 